\title{
ADUBAÇÃO FOSFATADA E METAIS PESADOS EM LATOSSOLO CULTIVADO COM ARROZ
}

\author{
Mônica Sartori de Camargo ${ }^{1 *}$; Ana Rosa Martins dos Anjos ${ }^{1}$; Carla Rossi ${ }^{1}$; Eurípedes Malavolta² \\ ${ }^{1}$ Pós-Graduandas do Depto. de Solos e Nutrição de Plantas - USP/ESALQ, C.P. 9 - CEP: 13418-900 - Piracicaba, SP. \\ USP/CENA, C.P. 96 - CEP: 13400-970 - Piracicaba, SP. \\ *Autor correspondente <mscamarg@carpa.ciagri.usp.br.>
}

RESUMO: A avaliação da contaminação por metais pesados contidos nos fertilizantes tem atraído a atenção devido a um eventual efeito no ambiente. O objetivo do trabalho foi estudar a absorção de metais pesados presentes nos adubos fosfatados pelo arroz, utilizando-se uma metodologia de extração seqüencial passível de ser adaptada à análise de rotina. O delineamento experimental foi em blocos casualizados e utilizaram-se quatro fontes de $\mathrm{P}$ (superfosfato triplo, termofosfato magnesiano e fosfatos naturais da Carolina do Norte e de Arad) em três doses (50, 100 e $200 \mathrm{mg} \mathrm{dm}^{-3}$ ). Foram cultivadas plantas de arroz após o cultivo de Brachiaria decumbens e Centrosema pubescens em Latossolo Vermelho-Amarelo distrófico, em condições de casa-devegetação. A transferência de metais pesados $(\mathrm{Cu}, \mathrm{Mn}, \mathrm{Cd}, \mathrm{Ni}, \mathrm{Zn})$ para o solo e para as plantas ocorreu de forma diferenciada para as doses e fontes utilizadas. Os adubos fosfatados não contribuíram de modo relevante para o aumento do teor de metais pesados no solo e nas plantas. $\mathrm{Na}$ extração seqüencial, o $\mathrm{HCl} 6 \mathrm{~mol} \mathrm{~L}^{-1}$ extraiu maior percentual de metais pesados do solo, seguido por Mehlich 1 e $\mathrm{H}_{2} \mathrm{O}$, exceto para Zn.

Palavras-chave: extratores, fósforo, fertilizantes

\section{PHOSPHATE FERTILIZERS AND HEAVY METALS IN AN OXISOL CULTIVATED WITH RICE}

\begin{abstract}
The contribuition of phosphate fertilizers to the pool of heavy metals in the soil and their transfer to plants and animals is a subject of concern specially among envirommentalists. The objective of this study was to assess the accumulation of heavy metals contained in phosphate fertilizers in the rice plant using a simple sequential method of extraction which could provide information about the availability of heavy metals in the soil. Greenhouse and laboratory experiments used a Xantic Ferralsol previously cultivated with Centrosema pubescens and Brachiaria decubens. The forages received triple superphosphate, termophosphate and North Caroline and Arad rock phosphates at the rates of 50,100 and $200 \mathrm{mg} \mathrm{dm}^{-3} \mathrm{P}$. There was no increase in heavy metals $(\mathrm{Cu}, \mathrm{Mn}, \mathrm{Cd}, \mathrm{Ni}, \mathrm{Zn})$ in the soil or in plants due to the rates of phosphate fertilizers applied. As expected, $6 \mathrm{~mol} \mathrm{~L}^{-1} \mathrm{HCl}$ extracted higher amounts of heavy metals than Mehlich 1 and water extractant.
\end{abstract}

Key words: extractants, phosphorus, fertilizers

\section{INTRODUÇÃO}

Metais pesados como $\mathrm{Cd}, \mathrm{Cr}, \mathrm{Pb}$ e $\mathrm{Hg}$, incluindose os micronutrientes $\mathrm{Fe}$, Cu e Mn podem ser introduzidos na cadeia alimentar pela adição de fertilizantes, principalmente fosfatados (Sharpley \& Menzel, 1987). Isso é resultado da ocorrência natural de vários metais pesados em rochas fosfáticas e de não serem eliminados no processo de manufatura. A contaminação do solo por metais pesados provocada pela aplicação de fertilizantes parece pequena, mas requer um monitoramento, pois seu uso é mais amplamente disseminado que outros agroquímicos (Amaral Sobrinho et al., 1996)

A determinação do teor de metais pesados em solos pode ser realizada pelo conteúdo total do elemento no solo, fração solúvel e trocável. O conteúdo total do elemento fornece o conhecimento da sua reserva no solo; a fração trocável é uma medida mais direta da probabilidade de eventuais efeitos prejudiciais ao ambiente.
Segundo Bertoncini (1997), a indefinição na escolha de métodos para extração dos teores totais e solúveis de metais em solos torna imprecisa a previsão do comportamento destes no ambiente e, consequentemente sua absorção pelas plantas.

A absorção é governada por vários processos. A sua complexidade pode explicar os resultados variados encontrados por diversos autores em relação à capacidade de alguns extratores químicos de prever a disponibilidade de metais pesados. Assim, o entendimento do comportamento dos metais no sistema solo-planta é importante na definição de métodos químicos mais eficientes (Gomes, 1996).

Duas diferentes técnicas de extração (simples e seqüencial) podem ser usadas para predizer a transferência dos metais pesados do solo para a cadeia alimentar. A técnica de extração simples consiste na determinação das diversas frações de metais pesados no solo de forma isolada usando amostras de diferentes solos. 
A extração seqüencial utiliza uma mesma amostra para fornecer informações sobre as frações químicas e a proporção do metal que é solubilizado por reagentes específicos, possibilitando a utilização de menor número de amostras para obtenção dos dados (Roca \& Pomares, 1991). A metodologia de extração sequencial possui alta viabilidade para tornar-se rotina no laboratório seja pelo trabalho com menor número de amostras e a maior confiabilidade nos resultados.

Geralmente, os parâmetros para o controle da poluição do solo baseiam-se na quantidade total de metais pesados. A fração total de um metal no solo, no entanto, não é um parâmetro que expresse sua disponibilidade para as plantas. Dentre os requisitos mínimos para ser ter uma boa metodologia e técnica de análise de solo encontra-se a reprodutibilidade, o baixo custo e a facilidade de adaptação aos procedimentos de rotina com uma alta correlação entre o teor de metal extraído e o absorvido pela planta (Roca \& Pomares, 1991).

Portanto, os objetivos do trabalho são estudar a transferência de metais pesados presentes nos adubos fosfatados para o arroz em condições de casa-devegetação; desenvolver uma metodologia preliminar de extração seqüencial passível de ser adaptada à análise de rotina.

\section{MATERIAL E MÉTODOS}

O experimento foi conduzido em casa-devegetação no Centro de Energia Nuclear na Agricultura (CENA/USP), com início em março de 1997. Utilizaramse amostras de um Latossolo Vermelho-Amarelo distrófico, textura média, coletado no Instituto de Zootecnia localizado no município de Nova Odessa - SP. As características químicas iniciais do solo se encontram na TABELA 1.
O solo estudado já havia sido submetido a dois cultivos anteriores, sendo o primeiro com centrosema (Moreira, 1997) e o segundo com braquiária. No primeiro cultivo, foi efetuada a calagem, a adubação de NPK e micronutrientes, sendo o delineamento utilizado inteiramente casualizado, em fatorial $4 \times 4 \mathrm{com} 3$ repetições. Os tratamentos do presente trabalho constituíram-se por quatro doses de fósforo $(0,50,100$ e $200 \mathrm{mg} \mathrm{P} \mathrm{dm}^{-3}$ ) e quatro fontes do nutriente (superfosfato triplo, ST, termofosfato magnesiano, TY, fosfato natural da Carolina do Norte, FNC e fosfato natural de Arad, FA) cujas características encontram-se na TABELA 2. O tratamento testemunha foi o mesmo para todas as fontes. A solução de micronutrientes aplicada durante o primeiro plantio teve a seguinte composição em mg kg-1: 0,5 de B; 0,1 de Co; 1,5 de $\mathrm{Cu} ; 5$ de $\mathrm{Fe} ; 5$ de Mn; 5 de $\mathrm{Zn}$ e 0,1 de Mo. No $2^{\circ}$ cultivo, o solo recebeu uma adubação de $150 \mathrm{mg} \mathrm{dm}^{-3}$ de $\mathrm{N}$ e K, dividida em três aplicações.

No presente trabalho, as unidades experimentais foram as mesmas dos trabalhos anteriores. Cada unidade experimental (vasos de $5 \mathrm{~L}$ ) recebeu $50 \mathrm{~mL}$ de uma solução contendo $3 \mathrm{~g}$ de $\mathrm{NH}_{4} \mathrm{NO}_{3}$ e $2 \mathrm{~g}$ de $\mathrm{KCl} \mathrm{L}^{-1}$, após o qual procedeu-se ao plantio de 10 sementes de arroz - IAC 165.

Após a germinação, foi realizado o desbaste para seis plantas por vaso e, diariamente, procedeu-se à irrigação mantendo o solo com $60 \%$ do poder de embebição. Aos 30 dias após a germinação, foram colhidos metade do número de plantas de cada vaso para posterior análise e efetuado o fornecimento de $\mathrm{N}$ em cobertura sob a forma de $\mathrm{NH}_{4} \mathrm{NO}_{3}$, o que foi repetido aos 60 dias.

Os metais pesados $\mathrm{Cu}, \mathrm{Fe}, \mathrm{Mn}, \mathrm{Zn}, \mathrm{Ni}$ e $\mathrm{Cd}$ contidos no solo foram analisados pela extração seqüencial, utilizando-se como extratores $\mathrm{H}_{2} \mathrm{O}$, Mehlich 1

TABELA 1 - Características químicas do solo utilizado.

\begin{tabular}{|c|c|c|c|c|c|c|c|c|c|c|}
\hline $\begin{array}{l}\mathrm{pH} \\
\mathrm{CaC}\end{array}$ & M.O & $\mathrm{K}$ & $\mathrm{Ca}$ & $\mathrm{Mg}$ & $\mathrm{Al}$ & $\mathrm{H}+\mathrm{Al}$ & SB & $\mathrm{T}$ & V & $\mathrm{m}$ \\
\hline & -- g dm $\mathrm{dm}^{3}$-- & - & --- & --- & $\mathrm{cmol}_{\mathrm{c}}$ & --.-- & --.-. & ---- & \multicolumn{2}{|c|}{-1} \\
\hline 3,7 & 20,3 & 0,1 & 0,3 & 0,2 & 2,1 & 9,6 & 0,6 & 10,2 & 6 & 78 \\
\hline $\mathrm{P}$ & & $\mathrm{S}-\mathrm{SO}_{4}^{-2}$ & & & $\mathrm{Cu}$ & \multicolumn{2}{|c|}{$\mathrm{Fe}$} & $\mathrm{Mn}$ & \multicolumn{2}{|c|}{$\mathrm{Zn}$} \\
\hline \multicolumn{11}{|c|}{--------- $\mathrm{mg} \mathrm{dm}^{3}$--------- } \\
\hline 4,0 & & 59,5 & \multicolumn{2}{|c|}{0,43} & 0,36 & \multicolumn{2}{|c|}{57} & 6,8 & \multicolumn{2}{|c|}{0,4} \\
\hline
\end{tabular}

TABELA 2 - Teores médios de fósforo e de metais pesados nos fertilizantes fosfatados.

\begin{tabular}{|c|c|c|c|c|c|c|c|c|c|c|}
\hline \multirow{3}{*}{ Fertilizantes } & \multicolumn{4}{|c|}{ Fósforo } & \multirow[b]{2}{*}{$\mathrm{Cd}$} & \multirow[b]{2}{*}{$\mathrm{Ni}$} & \multirow[b]{2}{*}{$\mathrm{Fe}$} & \multirow[b]{2}{*}{$\mathrm{Cu}$} & \multirow[b]{2}{*}{$\mathrm{Mn}$} & \multirow[b]{2}{*}{$\mathrm{Zn}$} \\
\hline & Total & Água & Ác cít. $2 \%$ & $\mathrm{CNA}+\mathrm{A} g u a$ & & & & & & \\
\hline & \multicolumn{10}{|c|}{ |- } \\
\hline Superf. triplo (ST) & 208 & 962 & 988 & 1055 & 1,7 & 785,3 & 1063,3 & 8,9 & 163,6 & 8,4 \\
\hline Termof. magnesiano (TY) & 396 & ----- & 371 & 171 & 37,2 & 589 & 1529,9 & 51,4 & 2613,5 & 494,9 \\
\hline Carolina Norte (FCN) & 663 & ----- & 213 & 122 & 46,6 & 33,5 & 1023,8 & 6,6 & 17,4 & 404,0 \\
\hline Fosfato Arad (FA) & 757 & ---- & 228 & 67 & 17,5 & 58,5 & 309,6 & 19,2 & ----- & 493,1 \\
\hline
\end{tabular}

Scientia Agricola, v.57, n.3, p.513-518, julho/set. 2000 
e $\mathrm{HCl} 6 \mathrm{~mol} \mathrm{~L}^{-1}$ e determinados por espectrometria de absorção atômica. Pesou-se inicialmente $5 \mathrm{~cm}^{3}$ de solo em um frasco de extração, ao qual se adicionou $40 \mathrm{~mL}$ de água deionizada, agitando-se por 30 minutos. Após um período de sedimentação de 15 minutos, o sobrenadante foi filtrado e utilizado para determinar teores de metais pesados extraídos em água. O material sedimentado no papel de filtro foi lavado com $40 \mathrm{~mL}$ da solução extratora Mehlich 1 e agitado por 30 minutos. O período de sedimentação foi o mesmo utilizado anteriormente e o filtrado foi utilizado para determinar os metais pesados extraídos em Mehlich 1. Da mesma forma como foi descrito anteriormente, $40 \mathrm{~mL}$ de uma solução $\mathrm{HCl} 6 \mathrm{~mol} \mathrm{~L}^{-1}$ foi utilizada para lavar o sedimento restante no papel, procedendo-se a seguir agitação, sedimentação e filtragem, sendo efetuada a determinação dos teores de metal pesado solúveis em $\mathrm{HCl} 6 \mathrm{~mol} \mathrm{~L}^{-1}$.

$\mathrm{Na}$ parte aérea, os teores de $\mathrm{Cu}, \mathrm{Fe}, \mathrm{Mn}, \mathrm{Zn}, \mathrm{Ni}$ e $\mathrm{Cd}$ foram analisados no extrato nítrico-perclórico, segundo metodologia descrita por Malavolta et al. (1989), determinados por espectrofotometria de absorção atômica.

\section{RESULTADOS E DISCUSSÃO}

\section{METAIS PESADOS NO SOLO}

Os teores encontrados nos fertilizantes utilizados (TABELA 2) estão em concordância com a literatura nacional, sendo menores, inclusive, para quase todos os nutrientes. Amaral Sobrinho et al. (1992) apresenta teores médios comumente encontrados nos fertilizantes comercializados no Brasil como para ST: 6565 ; 300; 24; 810 e 4,4 de $\mathrm{Fe}, \mathrm{Mn}, \mathrm{Ni}, \mathrm{Zn}$ e Cd, respectivamente; no TY, os teores de $\mathrm{Cu}, \mathrm{Fe}, \mathrm{Mn}, \mathrm{Ni}, \mathrm{Zn}$ e Cd são 44; 38410; 2220; 3300; 374 e 3,1 $\mathrm{mg} \mathrm{kg}^{-1}$.

Comparando-se os teores de metais pesados entre os extratores, foi observada diferenca, sendo que na extração seqüencial o $\mathrm{HCl} 6 \mathrm{~mol} \mathrm{~L}^{-1}$ extraiu maior percentual de $\mathrm{Fe}, \mathrm{Mn}, \mathrm{Cu}, \mathrm{Cd}$ e Ni, seguido de Mehlich 1 e água (Figura 1). Isso decorre de diferenças entre o modo de atuação dos extratores. A água extrai somente os nutrientes contidos na solução do solo. $O$ aumento observado nos teores de metais pesados recuperados pelo extrator Mehlich 1 indica que esta fração estaria disponível às plantas (Amaral Sobrinho et al., 1996). Já os teores

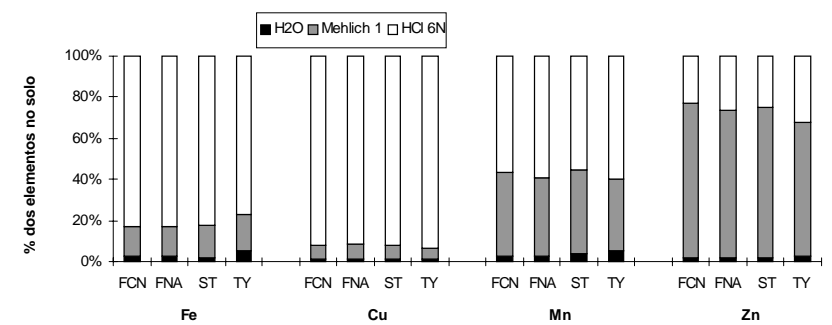

Figura 1 - Distribuição de Fe, Mn, Cu e Zn no solo em função dos diferentes extratores.

${ }^{*} \mathrm{FCN}=$ Fosfato da Carolina do Norte; FA=Fosfato de Arad; $\mathrm{ST}=$ superfosfato triplo; $\mathrm{TY}=$ termofosfato magnesiano. removidos pelo extrator $\mathrm{HCl} 6 \mathrm{~N}$ estão relacionados à energia desse extrator, que não diferencia entre as várias categorias de minerais presentes no solo. Assim, considera-se que o $\mathrm{HCl}$ possui a capacidade de extrair nutrientes restantes, que não se encontram prontamente disponíveis às plantas (Piper, 1944).

A única exceção a este comportamento foi o $\mathrm{Zn}$, que apresentou teores maiores no solo extraídos pelo extrator Mehlich 1 que para $\mathrm{HCl} 6 \mathrm{~mol} \mathrm{~L}^{-1}$. Isto pode ser conseqüência do material de origem deste solo, que possivelmente tem teores naturais extremamente baixos de $\mathrm{Zn}$, restando, assim, menor quantidade de $\mathrm{Zn}$ para ser extraída pelo $\mathrm{HCl}$. As quantidades de $\mathrm{Cd}$ e Ni presentes na análise foram extremamente reduzidas, próximas a zero, não sendo possível sua representação gráfica.

\section{COMPARAÇÃO ENTRE OS MÉTODOS}

Não foi constatada tendência definida para a interação entre doses e fontes. O efeito das doses dos fertilizantes sobre os teores de metais extraídos do solo não foi consistente, sendo realizada apenas comparação entre as fontes de fósforo (TABELA 3).

Para o extrator $\mathrm{H}_{2} \mathrm{O}$, foi verificado efeito de fontes somente para os teores de $\mathrm{Fe}$ e $\mathrm{Cu}$, sendo os valores sempre superiores para o TY (TABELA 3). Isso se deve, provavelmente, ao seu alto conteúdo no fertilizante (TABELA 2). Os metais pesados cujos teores são encontrados em baixa quantidade no solo como $\mathrm{Cd}$ e $\mathrm{Ni}$ não mostraram teores relevantes no solo ao serem extraídos pela água. Isso já havia sido constatado por Haq et al. (1980) em 46 solos devido ao seu baixo conteúdo no solo e ao modo de ação desse extrator.

Quanto ao extrator Mehlich 1, ocorreu o contrário, pois apenas os teores de ferro e manganês não tiveram diferença entre as fontes, o que pode ser devido à sua grande quantidade nos solos tropicais, reduzindo o efeito das fontes (TABELA 3). Os teores de ferro e manganês estão compatíveis com os citados na literatura (Sillanpaa, 1982).

À semelhança do ocorrido com $\mathrm{H}_{2} \mathrm{O}$, as determinações de metais pesados em Mehlich 1 não mostraram teores relevantes de $\mathrm{Cd}$ e $\mathrm{Ni}$ no solo, devido, provavelmente, à baixa quantidade no solo. $\mathrm{O}$ teor de $\mathrm{Zn}$ para $T Y$ foi significativamente menor $\left(2,11 \mathrm{mg} \mathrm{dm}^{-3}\right)$ que as demais, apesar do baixo teor de Zn no ST (TABELA 3). Camargo et al. (1982) observaram teores de $\mathrm{Zn}$ em solos de São Paulo, variando de 0,6 a $13 \mathrm{mg} \mathrm{dm}^{-3}$, nos quais estão incluídos o do experimento. E para os teores de $\mathrm{Cu}$, os valores estão compreendidos dentro de uma faixa de valores comumente encontrados em solos brasileiros (Malavolta, 1994).

Para metais pesados extraídos por $\mathrm{HCl} 6 \mathrm{~mol} \mathrm{~L}^{-1}$, o ferro foi o único sem diferença entre as fontes devido possivelmente ao seu alto teor no solo, sendo os teores médios totais encontrados variando de 478 a $500 \mathrm{mg} \mathrm{dm}^{-3}$. 
TABELA 3 - Teores de metais pesados no solo extraídos por $\mathrm{H}_{2} \mathrm{O}$, Mehlich1 e $\mathrm{HCl} 6$ mol L-1, para as diferentes fontes, dentro de doses de $P$.

\begin{tabular}{|c|c|c|c|c|c|c|}
\hline \multirow{2}{*}{$\begin{array}{l}\text { Fertilizantes } \\
\text { Fosfatados }\end{array}$} & \multicolumn{6}{|c|}{$\mathrm{H}_{2} \mathrm{O}$} \\
\hline & $\mathrm{Fe}$ & $\mathrm{Cu}$ & $\mathrm{Mn}$ & $\mathrm{Cd}$ & $\mathrm{Ni}$ & $\mathrm{Zn}$ \\
\hline & --------- & - & ------- n & 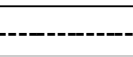 & ----- & - \\
\hline FCN & $14,5 b$ & $0,32 \mathrm{~b}$ & 3,8 & 0,00 & 0,00 & 0,07 \\
\hline FA & $17,6 \mathrm{~b}$ & $0,36 \mathrm{~b}$ & 2,6 & 0,00 & 0,00 & 0,07 \\
\hline ST & $11,0 \mathrm{~b}$ & $0,39 \mathrm{~b}$ & 2,7 & 0,00 & 0,00 & 0,07 \\
\hline \multirow[t]{3}{*}{ TY } & $34,2 \mathrm{a}$ & $0,58 \mathrm{a}$ & 2,9 & 0,00 & 0,03 & 0,09 \\
\hline & & \multicolumn{5}{|c|}{ Mehlich } \\
\hline & $\mathrm{Fe}$ & $\mathrm{Cu}$ & $\mathrm{Mn}$ & $\mathrm{Cd}$ & $\mathrm{Ni}$ & $\mathrm{Zn}$ \\
\hline FCN & $84,27 \mathrm{a}$ & $5,12 \mathrm{a}$ & $16,95 \mathrm{a}$ & $0,000 \mathrm{~b}$ & $0,000 \mathrm{~b}$ & $2,59 \mathrm{a}$ \\
\hline FA & 85,77 a & $4,92 a b$ & $16,62 \mathrm{a}$ & $0,000 \mathrm{~b}$ & $0,004 \mathrm{~b}$ & $2,51 a b$ \\
\hline ST & $94,96 \mathrm{a}$ & $4,11 \mathrm{bc}$ & $17,48 \mathrm{a}$ & $0,000 \mathrm{~b}$ & $0,000 \mathrm{~b}$ & $2,48 a b$ \\
\hline \multirow[t]{3}{*}{ TY } & 93,73 a & $3,96 \mathrm{c}$ & $17,04 a$ & $0,015 \mathrm{a}$ & $0,035 a$ & $2,11 \mathrm{~b}$ \\
\hline & & \multicolumn{5}{|c|}{$\mathrm{HCl}$} \\
\hline & $\mathrm{Fe}$ & $\mathrm{Cu}$ & $\mathrm{Mn}$ & $\mathrm{Cd}$ & $\mathrm{Ni}$ & $\mathrm{Zn}$ \\
\hline FCN & $95,67 \mathrm{a}$ & $7,06 a b$ & $248,00 \mathrm{~b}$ & $0,685 \mathrm{~b}$ & $0,683 \mathrm{~b}$ & $0,809 a$ \\
\hline FA & $100,14 a$ & $7,61 \mathrm{a}$ & $200,60 \mathrm{c}$ & $0,775 \mathrm{a}$ & $0,665 \mathrm{bc}$ & $0,927 \mathrm{a}$ \\
\hline ST & $99,44 \mathrm{a}$ & $5,54 \mathrm{c}$ & 227,56 b & $0,493 d$ & $0,541 \mathrm{c}$ & $0,861 \mathrm{a}$ \\
\hline TY & 99,63 a & $6,78 \mathrm{~b}$ & $274,68 a$ & $0,613 \mathrm{c}$ & $0,886 a$ & $1,195 \mathrm{a}$ \\
\hline
\end{tabular}

'Letras minúsculas iguais na coluna não diferem significativamente para as fontes a $5 \%$, pelo teste de Turkey.

${ }^{\star} \mathrm{FCN}=$ Fosfato da Carolina do Norte; FA=Fosfato de Arad; $\mathrm{ST}=$ superfosfato triplo; $\mathrm{TY}=$ termofosfato magnesiano

Para o Cu, os valores encontrados no solo estão dentro da faixa de variação para solos do Estado de São Paulo (6 a $46 \mathrm{mg} \mathrm{dm}^{-3}$ ) derivados de sedimentos modernos, entre os quais se incluem Latossolos VermelhoAmarelos (Malavolta,1994). Os resultados apresentados indicam que menores teores totais de $\mathrm{Cu}$ foram encontrados em tratamentos com o ST. Comparando-se estes teores com os adicionados ao solo em função da composição nos fertilizantes, pode-se afirmar que os mesmos estão condizentes com o baixo teor de Cu encontrado no ST. Observa-se, contudo, que apesar do FCN ter apresentado baixo conteúdo de Cu em sua composição, os teores encontrados no solo foram semelhantes aos outros com maior teor. Isso pode ser devido à sua lenta disponibilização no solo, restando maior quantidade no solo comparado aos outros. (TABELA 3).

Quanto ao $\mathrm{Mn}$, os teores foram mais elevados para TY devido ao seu alto teor no fertilizante. O FCN, no entanto, apresentou teores acima do esperado, o que pode ser decorrência de sua origem (TABELA 3).

Quanto aos teores totais de Cd no solo, os mesmos encontram-se mais baixos para a fonte fosfatada ST, que foi o fertilizante que apresentou teor mais baixo no fertilizante. Os fosfatos naturais (Carolina do Norte e Arad), por sua vez, tiveram os maiores teores, sendo similares entre si. Estes teores encontrados no presente estudo localizam-se muito abaixo da faixa considerada crítica (3 a $8 \mathrm{mg}$ Cd total $\mathrm{dm}^{-3}$ de solo) por Kabata-Pendias
\& Pendias (1984) e confirmam resultados de diversos experimentos a nível mundial, no qual não foram encontrados aumentos relevantes de $\mathrm{Cd}$ no solo devido à utilização de adubações fosfatadas intensas e freqüentes durante vários anos (Mulla et al., 1980; Mortved, 1987).

As parcelas que receberam aplicações de TY, mostraram sempre conteúdo de Ni mais elevado devido ao seu alto teor no fertilizante. Esse teor relativamente alto do Ni é consequência da matéria-prima usada na produção (rocha fosfática e a serpentina do aparelho utilizado para concentração de $\mathrm{P}$ ).

O comportamento do $\mathrm{Zn}$ no solo apresentou variações significativas entre as fontes utilizadas. Para os fosfatos de rocha reativos (FCN e FA), os teores de $\mathrm{Zn}$ foram inferiores aos dos tratamentos com as fontes solúveis. Mandal \& Mandal (1990), estudando o efeito da aplicação de $\mathrm{P}$ nas transformações da fração $\mathrm{Zn}$ no solo e na nutrição de arroz, relatou redução no teor de $Z n$ total do solo (Método DBC) à medida que se aumentaram as doses de $\mathrm{P}$ aplicadas.

\section{METAIS PESADOS NA PLANTA}

$\mathrm{Na}$ planta, houve interação significativa entre fontes e doses de fertilizantes fosfatados para Fe, Mn e Zn (TABELA 4). Para Cu e Zn, houve efeito somente de doses. As equações de regressão obtidas foram $\mathrm{Cr}=$ $14,54-0,077 X-0,0002 X^{2} r^{2}=0,75 ; Z n=1,63-0,0038 X$ 
TABELA 4 - Teores de metais pesados na planta para as diferentes fontes, dentro de doses de P.

\begin{tabular}{|c|c|c|c|c|c|c|c|c|c|}
\hline & \multicolumn{3}{|c|}{$\mathrm{Fe}$} & \multicolumn{3}{|c|}{$\mathrm{Mn}$} & \multicolumn{3}{|c|}{$\mathrm{Zn}$} \\
\hline & 50 & 100 & 200 & 50 & 100 & 200 & 50 & 100 & 200 \\
\hline & . & & & 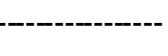 & $\mathrm{ng} \mathrm{kg}^{-1}$ & & & & \\
\hline FCN & $176,86 \mathrm{c}$ & $206,25 \mathrm{c}$ & $284,15 \mathrm{a}$ & $915,90 \mathrm{~b}$ & $814,30 \mathrm{~b}$ & $547,00 \mathrm{~b}$ & $168,32 \mathrm{a}$ & $181,98 \mathrm{a}$ & $163,16 \mathrm{a}$ \\
\hline FA & $129,92 \mathrm{c}$ & $141,87 \mathrm{c}$ & $167,53 \mathrm{a}$ & $1031,30 \mathrm{~b}$ & $904,60 \mathrm{~b}$ & $668,70 \mathrm{~b}$ & $180,30 \mathrm{a}$ & 184,48 a & $177,94 \mathrm{a}$ \\
\hline ST & $620,02 \mathrm{a}$ & $415,23 \mathrm{~b}$ & $285,55 \mathrm{a}$ & $1612,30 \mathrm{a}$ & $1792,90 \mathrm{a}$ & $1716,40 a$ & $174,17 \mathrm{a}$ & 189,78 a & $166,37 \mathrm{a}$ \\
\hline TY & $455,35 \mathrm{~b}$ & $530,35 a$ & $230,50 a$ & $925,90 \mathrm{~b}$ & $484,30 \mathrm{~b}$ & $121,70 \mathrm{c}$ & $180,44 \mathrm{a}$ & $146,72 \mathrm{~b}$ & $82,48 \mathrm{~b}$ \\
\hline
\end{tabular}

${ }^{1}$ Dentro de cada dose, letras minúsculas iguais na coluna não diferem significativamente para as fontes a Duncan $5 \%$. OBS: Para a dose 0, não houve diferença estatística, em função de os resultados serem os mesmos para todas as fontes.

${ }^{2} \mathrm{FCN}=$ Fosfato da Carolina do Norte; FA=Fosfato de Arad; ST= superfosfato triplo; TY= termofosfato magnesiano

$r^{2}=0,89$. O Cd não apresentou resposta nem a fontes e doses.

As altas concentrações de Mn na planta de arroz, segundo Takahashi (1995), Obata (1995) e Marschner (1995), ao contrário de outras plantas, não acarretam em toxidez deste elemento. Isso ocorre devido ao acúmulo de silício nesta cultura, que pode chegar a níveis de $12 \%$, possibilitar uma melhor distribuição do Mn na planta. Segundo Obata (1995), teores de Mn nas lâminas foliares em torno de $2.000 \mathrm{mg} \mathrm{kg}^{-1}$, que foram observados em solos que apresentavam até $300 \mathrm{mg} \mathrm{dm}^{-3}$, não afetam o crescimento do arroz, embora diminuam o rendimento de grãos.

As concentrações médias de $\mathrm{Zn}, \mathrm{Cu}, \mathrm{Ni}$ e $\mathrm{Cd}$ no tecido foram respectivamente: $180,24,56,2,88$ e 0,19 $\mathrm{mg} \mathrm{kg}{ }^{-1}$, concordando com Haq et al. (1980) que encontraram teores bastante variáveis de metais pesados em Beta vulgaris, os quais foram em média: 220 de $\mathrm{Zn}$; 1,55 de Cd; 4,6 de Ni e $19 \mathrm{mg} \mathrm{kg}^{-1}$ de Cu.

Silva (1994), estudando metais pesados em milho cultivados com diferentes fontes e doses de $\mathrm{P}$, observou os seguintes teores médios na parte aérea: $0,4 \mathrm{mg} \mathrm{kg}^{-1}$ de Cd; 44, $5 \mathrm{mg} \mathrm{kg}^{-1}$ de Zn.

Obata (1995) relata que o arroz apresenta em média teores de Cu aproximados de 5 a $20 \mathrm{mg} \mathrm{kg}^{-1}$ e que excesso de Cu no solo somente causa dano quando o teor solúvel deste elemento for maior que $125 \mathrm{mg} \mathrm{dm}^{-3}$. O mesmo autor indica que os teores normais de $\mathrm{Zn}$ em arroz situam-se entre 30 a $100 \mathrm{mg} \mathrm{kg}^{-1}$, enfatizando ser esta espécie relativamente tolerante ao seu excesso. Se ocorrer a toxidez, ela aparece na forma de um decréscimo na altura da planta e no número de perfilhos.

As análises de correlação entre teor de metais pesados no solo por diferentes extratores, teor do metal pesado na planta e a produção de matéria seca do arroz não foram significativas, à exceção do $\mathrm{Cd}\left(r^{2}=0,70\right)$. Do mesmo modo, Haq et al. (1980) citam que o crescimento da planta não foi afetado pelos metais estudados e a produção de matéria seca não foi significativamente correlacionada com as concentrações dos metais nas plantas. Outrossim, Haq \& Miller (1972) relatam não haver correlação entre os teores de $\mathrm{Mn}$ e Cu no solo e os teores destes absorvidos pelo milho.

Quanto ao Cu, Alloway (1995) cita que a absorção de Cu pelas plantas não se correlaciona bem com as quantidades existentes na reserva do solo e/ou com quantidades removidas pelos diversos extratores devido à sua atividade química não ser constante na solução do solo. Reisenauer, citado por Alloway (1995), conclui que as análises de solo não correlacionaram bem com a absorção pela planta também para o $\mathrm{Mn}$ devido às mudanças na rizosfera que podem reduzir ou aumentar a solubilidade de $\mathrm{Mn}$ no solo e sua aquisição pelas raízes. Para o Ni, entretanto, Alloway (1995) relata que a concentração deste nas plantas geralmente reflete a concentração do elemento no solo, especialmente com a fração solúvel .

Portanto, que os metais pesados, micronutrientes ou não, contidos nos adubos comerciais, principalmente fosfatados, usados nas doses adequadas, não elevam os teores destes elementos nos solos e nas plantas a níveis indesejáveis em prazos curto, médio e longo concordando com Malavolta (1994).

\section{CONCLUSÕES}

- Os adubos fosfatados não contribuíram de modo relevante para o aumento do teor de metais pesados no solo e na planta.

- Na extração seqüencial, o $\mathrm{HCl} 6 \mathrm{~mol} \mathrm{~L}^{-1}$ extraiu maior percentual de metais pesados do solo seguido por Mehlich 1 e $\mathrm{H}_{2} \mathrm{O}$, exceto para $\mathrm{Zn}$.

\section{REFERÊNCIAS BIBLIOGRÁFICAS}

ALLOWAY, B.J. Heavy metals in soils. London: Blackie Academic \& Profesional, 1995. 366p.

AMARAL SOBRINHO, N.M.B.; BARROS, N.F.; COSTA, L.M.; FONTES, M.P.F. Efeito de um resíduo da indústria de $\mathrm{Zn}$ sobre a química de amostras de solo e plantas de milho. Revista Brasileira de Ciência do Solo, v.20, p.271-276, 1996. 
AMARAL SOBRINHO, N.M.B.; COSTA, L.M.; OLIVEIRA, C.; VELLOSO, A.C.X. Metais pesados em alguns fertilizantes e corretivos. Revista Brasileira de Ciência do Solo, v.16, p.271-276, 1992.

CAMARGO, O.A.; VALADARES, J.M.A.S.; DECHEN, A.R Efeitos do $\mathrm{pH}$ e da incubação na extração do manganês, zinco, cobre e ferro no solo. Revista Brasileira de Ciência do Solo, v.16, p.83-88, 1982.

BERTONCINI, E.I. Mobilidade de metais pesados em solos tratados com solo de esgoto. Piracicaba, 1997. 90p. Dissertação (Mestrado) - Escola Superior de Agricultura Luiz de Queiroz, Universidade de São Paulo.

GOMES, P.C. Fracionamento e biodisponibilidade de metais pesados influenciados por calagem e concentrações de metais em Latossolo Vermelho-Amarelo. Viçosa, 1996. 161p. Tese (Doutorado) - Universidade Federal de Viçosa.

HAQ, A.U.; BATES, T.E.; SOON, Y.K. Comparison of extractants of plant-available $\mathrm{Zn}, \mathrm{Cd}, \mathrm{Ni}$ e $\mathrm{Cu}$ in contaminated soils. Soil Science Society of America Journal, v.44, p.772-776, 1980.

HAQ, A.U.; MILLER, M.H. Prediction of available soil. Zn, Cu and Mn using chemical extractants. Agronomy Journal, v.64, p.779-782, 1972

KABATA-PENDIAS, A.; PENDIAS, $H$. Trace elements in soils and plants. Boca Raton: CRC Press, 1984. 315p.

MALAVOLTA, E. Fertilizantes e seu impacto ambiental: micronutrientes e metais pesados, mitos, mistificações e fatos. São Paulo: ProduQuímica, 1994. 153p.

MALAVOLTA, E.; VITTI, G.C.; OLIVEIRA, S.A. Avaliação do estado nutricional das plantas: princípios e aplicações. Piracicaba: Potafós, 1989. 201p.

MANDAL, B.; MANDAL, L.N. Effect of phosphorus application on transformations of zinc fraction in soil and on the zinc nutrition of lowland rice. Plant and Soil, v.121, p.115-123, 1990.

MARSCHNER, $\mathrm{H}$. Mineral nutrition of higher plants. 2.ed. London: Academic Press, 1995. 889p.

MOREIRA, A. Efeitos de fontes e doses de fósforo na alfafa (Medicago sativa L.) e centrosema (Centrosema pubescens) e avaliação de extratores. Piracicaba, 1997. 107p. Dissertação (Mestrado) - Escola Superior de Agricultura Luiz de Queiroz, Universidade de São Paulo.
MORTVED, J.J. Cadmium levels in soils and plants from some long term soil fertility experiments in the United States of America. Journal of Enviromental Quality, v.16, p.137-142, 1987.

MULLA, D.J.; PAGE, A.L.; GANJE, T.J. Cadmium accumulation and bioavailability in soils from long term phosphous fertilization. Journal of Enviromental Quality, v.9, p.408-412, 1980.

OBATA, $\mathrm{H}$. Micro Essential elements. In: MATSUO, T.; KUMAZAWA, K.; ISHII, R.; ISHIHARA, K.; HIRATA, H. (Ed.) Science of the rice plant. Tokyo: Food and agriculture Policy Research Center, 1995. v.2, p.420-433.

PIPER, C.S. The analysis of the hydrocloric acid extract. In: PIPER, C.S. Soil and plant analysis. New York: Interscience Publishers, 1944. cap.8, p.137-153.

ROCA, J.; POMARES, F. Prediction of available heavy metals by six chemical extractants in a sewage sludge-amended soil. Communications in Soil Science and Plant Analysis, v.22, p.2119-2136, 1991

SHARPLEY, A.N.; MENZEL, R.G. The impact of soil and fertilizer phosphorus on the environmment. Advances in Agronomy, v.41, p.297-324, 1987.

SILLANPAA, M. Micronutrient and the nutrient status of soils: a global study. Roma: FAO, 1982. 44p. (Soils Bulletin, 48)

SILVA, A.J. Metais pesados em plantas de milho (Zea mays L.) cultivadas com diferentes fontes e doses de fósforo, formas de localização de fertilizantes e níveis de calagem. Viçosa, 1994. 135p. Tese (Doutorado) - Universidade Federal de Viçosa.

TAKAHASHI, E. Uptake mode and physiological functions of silica. In: MATSUO, T.; KUMAZAWA, K.; ISHII, R.; ISHIHARA, K.; HIRATA, H. (Ed.) Science of the rice plant. Tokyo: Food and Agriculture Policy Research Center, 1995. v.2, p.402-417.

Recebido em 19.03.99 